PROCEEDINGS OF THE

AMERICAN MATHEMATICAL SOCIETY

Volume 134, Number 3, Pages 731-735

S 0002-9939(05)08019-6

Article electronically published on July 18, 2005

\title{
OPTIMAL WEYL INEQUALITY IN BANACH SPACES
}

\author{
AICKE HINRICHS \\ (Communicated by N. Tomczak-Jaegermann) \\ Dedicated to Professor Albrecht Pietsch on the occasion of his 70th birthday
}

AbStract. A well-known multiplicative Weyl inequality states that the sequence of eigenvalues $\left(\lambda_{k}(T)\right)$ and the sequence of approximation numbers $\left(a_{k}(T)\right)$ of any compact operator $T$ in a Banach space satisfy

$$
\prod_{k=1}^{n}\left|\lambda_{k}(T)\right| \leq n^{n / 2} \prod_{k=1}^{n} a_{k}(T)
$$

for all $n$. We prove here that the constant $n^{n / 2}$ is optimal, which solves a longstanding problem.

\section{INTRODUCTION}

Estimating the eigenvalue distribution of compact operators via approximation quantities is a classic theme in approximation theory with an extensive literature. The starting point was the inequality

$$
\prod_{k=1}^{n}\left|\lambda_{k}(T)\right| \leq \prod_{k=1}^{n} s_{k}(T)
$$

proved by $\mathrm{H}$. Weyl in [Wey49 for any compact operator $T$ acting on a Hilbert space. Here $\left(\lambda_{k}(T)\right)$ and $\left(s_{k}(T)\right)$ are the sequences of eigenvalues and singular numbers of $T$, respectively. A great deal of effort went into proving similar inequalities for operators on Banach spaces. For a recent survey article we refer to Koe01.

The most useful inequality for the investigation of the asymptotic behavior of the eigenvalue sequence is due to A. Pietsch [Pie80a]. It states that there exists some constant $c>1$ such that for any continuous linear operator $T$ on some Banach space and all $n=1,2, \ldots$,

$$
\prod_{k=1}^{n}\left|\lambda_{k}(T)\right| \leq c^{n} \prod_{k=1}^{n} \dot{x}_{k}(T)
$$

Here $\left(\dot{x}_{k}(T)\right)=\left(x_{1}(T), x_{1}(T), x_{2}(T), x_{2}(T), x_{3}(T), x_{3}(T), \ldots\right)$ is the doubled sequence of Weyl numbers of $T$. For the definition of Weyl numbers see below. This inequality is sufficient to prove that the eigenvalue sequence is in $l_{p}$ for some $p>0$

Received by the editors October 6, 2004.

2000 Mathematics Subject Classification. Primary 47B10, 43A25.

Key words and phrases. Weyl inequality, eigenvalue estimates, approximation numbers, $s$ numbers.

The research of the author was supported by the DFG Emmy-Noether grant Hi 584/2-3.

(C)2005 American Mathematical Society Reverts to public domain 28 years from publication 
provided that the sequence of Weyl numbers is in $l_{p}$. In particular, for any $p>0$ there exists a constant $c_{p}$ such that

$$
\left(\sum_{k=1}^{\infty}\left|\lambda_{k}(T)\right|^{p}\right)^{1 / p} \leq c_{p}\left(\sum_{k=1}^{\infty} x_{k}(T)^{p}\right)^{1 / p} .
$$

This is also due to A. Pietsch Pie80a].

Two obvious unaesthetic features of (2) compared with (1) are the appearance of the constant $c>1$ and the replacement of the sequence of singular numbers with the doubled sequence of Weyl numbers instead of the more natural original sequence of Weyl numbers. An example showing that $c>1$ is necessary in the general case was given in Koe86, 2.a.6]. Whether the doubled Weyl number sequence can be replaced by the usual sequence was an open problem (stated e.g. in [Koe86, Koe01, Pie87]), which we are going to solve in this note.

This is not merely an aesthetic problem for two reasons. First, it was shown by H. König in [Koe86, 2.a.7] that if inequality (2) would be true with $x_{k}(T)$ instead of $\dot{x}_{k}(T)$, then the constant $c_{p}$ in (3) could be chosen independently of $p>0$. The best known (for $p \rightarrow 0$ ) upper bound $c_{p} \leq 2 e / \sqrt{p}$ is also due to H. König Koe84. and tends to infinity as $p$ goes to 0 . Second, if one wants to estimate the first few eigenvalues of an operator, inequality (2) is practically of no help. Instead, one would need inequalities of the type

$$
\prod_{k=1}^{n}\left|\lambda_{k}(T)\right| \leq c_{n} \prod_{k=1}^{n} x_{k}(T)
$$

with optimal $c_{n}$. This inequality with $c_{n}=n^{n / 2}$ seems to be well known although it does not appear in print. We will give a proof of this inequality in due time. The main result of this note is that the constant $c_{n}=n^{n / 2}$ is actually optimal even if we allow the Weyl numbers to be replaced by the approximation numbers.

We now introduce the necessary notation. Following A. Pietsch Pie74, Pie80b, Pie87, a sequence $\left(s_{n}\right)_{n=1}^{\infty}$ of maps assigning to each operator $T \in \mathcal{L}(E, F)$ between Banach spaces $E$ and $F$ numbers $s_{n}(T)$ with the properties

(i) $\|T\|=s_{1}(T) \geq s_{2}(T) \geq \ldots \geq 0$ for $T \in \mathcal{L}(E, F)$,

(ii) $s_{m+n-1}(S+T) \leq s_{m}(S)+s_{n}(T)$ for $S, T \in \mathcal{L}(E, F)$,

(iii) $s_{n}(B T A) \leq\|B\| s_{n}(T)\|A\|$ for $A \in \mathcal{L}\left(E_{0}, E\right), T \in \mathcal{L}(E, F), B \in \mathcal{L}\left(F_{0}, F\right)$,

(iv) $s_{n}(T)=0$ if $\operatorname{rank} T<n$,

(v) $s_{n}\left(I_{n}\right)=1$ for the identitiy maps $I_{n}: l_{2}^{n} \rightarrow l_{2}^{n}$ on $l_{2}^{n}$

is called an $s$-number sequence. Moreover, $s_{n}(T)$ is called the $n$-th $s$-number of $T$. Basic examples for our purposes are the approximation numbers given by

$$
a_{n}(T):=\inf \{\|T-A\|: A \in \mathcal{L}(E, F), \operatorname{rank} A<n\},
$$

the Weyl numbers given by

$$
x_{n}(T):=\sup \left\{a_{n}(T A): A \in \mathcal{L}\left(l_{2}, E\right),\|A\| \leq 1\right\},
$$

and the Hilbert numbers given by

$$
h_{n}(T):=\sup \left\{a_{n}(B T A): A \in \mathcal{L}\left(l_{2}, E\right), B \in \mathcal{L}\left(F, l_{2}\right),\|A\|,\|B\| \leq 1\right\} .
$$

For compact operators on Hilbert spaces and any $s$-number sequence, $s_{n}(T)$ is just the $n$-th singular value of $T$; see [Pie87, 2.11.9]. The Hilbert numbers form the smallest $s$-number sequence, the approximation numbers the largest, i.e. $h_{n}(t) \leq$ 
$s_{n}(T) \leq a_{n}(T)$ for all operators $T$ and all $n$; see [Pie87, 2.3.4 and 2.6.3]. For a compact operator acting on some Banach space, $\left(\lambda_{n}(T)\right)$ denotes the sequence of eigenvalues of $T$ counted according to their multiplicity and arranged by nonincreasing modulus. For more information on $s$-numbers and their relations to eigenvalues we refer to Koe86, Koe01, Pie87.

Our main result is formulated in the following theorem.

Theorem 1.1. Let $\left(s_{n}\right)$ be an s-number sequence. Then

$$
\prod_{k=1}^{n}\left|\lambda_{k}(T)\right| \leq n^{n / 2} \prod_{k=1}^{n} s_{k}(T)
$$

for any compact operator $T$ on a Banach space and all $n$. The constant $n^{n / 2}$ in this inequality is optimal.

By the already mentioned result of H. König from [Koe86, 2.a.7] we have

Corollary 1.2. The constants $c_{p}$ in (3) cannot be chosen independent of $p>0$ even if the Weyl numbers in the inequality are replaced with the approximation numbers.

\section{THE PROOFS}

We start with the proof of the inequality in Theorem 1.1. Since the Hilbert numbers form the smallest $s$-scale, it is enough to consider the case $s_{k}(T)=h_{k}(T)$. If $\lambda_{n}(T)=0$, there is nothing to prove. So we assume $\lambda_{n}(T) \neq 0$. Then by Koe86, 1.a.4] or Pie87, 3.2.23] we can find an $n$-dimensional subspace $E_{n}$ of $E$ invariant under $T$ such that $T_{n}$ has the eigenvalues $\lambda_{1}(T), \ldots, \lambda_{n}(T)$. By a result of S. Kwapien we can find a projection $P_{n}$ from $E$ onto $E_{n}$ with 2-summing norm $\pi_{2}\left(P_{n}\right)=\sqrt{n}$. Now the Pietsch factorization theorem [Pie66, Pie80b] implies that $P_{n}$ can be factored as $P_{n}=B_{n} A_{n}$ with a norm 1 operator $B_{n} \in \mathcal{L}\left(l_{2}^{n}, E_{n}\right)$ and an operator $A_{n} \in \mathcal{L}\left(E, l_{2}^{n}\right)$ with $\left\|A_{n}\right\| \leq \pi_{2}\left(A_{n}\right)=\sqrt{n}$. Let $J_{n}$ be the embedding of $E_{n}$ into $E$. Applying Weyl's inequality to the Hilbert space operator $S_{n}=$ $A_{n} J_{n} T_{n} B_{n}=A_{n} T J_{n} B_{n}$ together with the principle of related operators (cf. Pie87, 3.3.4]) we obtain that

$$
\begin{aligned}
\prod_{k=1}^{n}\left|\lambda_{k}(T)\right| & =\prod_{k=1}^{n}\left|\lambda_{k}\left(T_{n}\right)\right|=\prod_{k=1}^{n}\left|\lambda_{k}\left(S_{n}\right)\right|=\prod_{k=1}^{n} h_{k}\left(S_{n}\right) \\
& \leq \prod_{k=1}^{n}\left\|A_{n}\right\| h_{k}(T)\left\|J_{n}\right\|\left\|B_{n}\right\| \leq n^{n / 2} \prod_{k=1}^{n} h_{k}(T) .
\end{aligned}
$$

For the proof of the optimality in Theorem 1.1, let $c_{n}$ denote the smallest possible constant in the inequality

$$
\prod_{k=1}^{n}\left|\lambda_{k}(T)\right| \leq c_{n} \prod_{k=1}^{n} a_{k}(T) .
$$

To show that $c_{n} \geq n^{n / 2}$, we need an auxiliary lemma.

Lemma 2.1. For any $n=1,2, \ldots$,

$$
\sup \frac{\prod_{k=1}^{n} \sigma_{k}}{\prod_{k=1}^{n} \sum_{l=k}^{n} \sigma_{l}}=1
$$

where the supremum is taken over all real $\sigma_{1}, \ldots, \sigma_{N}$ with $\sigma_{1}>\sigma_{2}>\ldots>\sigma_{n}>0$. 
Proof. Denote the supremum by $a_{n}$. Obviously, $a_{1}=1$. Now assume that $n>1$ and $a_{n-1}=1$ is already proved. Observe that

$$
a_{n}=\sup \frac{\prod_{k=1}^{n-1} \sigma_{k}}{\prod_{k=1}^{n-1} \sum_{l=k}^{n} \sigma_{l}}
$$

The expression in the supremum is a continuous nonincreasing function of $\sigma_{n}>0$, which is also continuous for $\sigma_{n}=0$. Hence the sup is attained for $\sigma_{n}=0$, which just gives $a_{n}=a_{n-1}=1$.

We now construct our example. Let $E_{n}=\left(e_{h k}\right)$ be the $n \times n$ Fourier matrix which has entries $e_{h k}=n^{-1 / 2} \exp (2 \pi i h k / n)$. Fix numbers $\sigma_{1}>\sigma_{2}>\ldots>\sigma_{n}>0$ and let $D$ be the diagonal matrix with diagonal $\left(\sigma_{1}, \ldots, \sigma_{n}\right)$. Let $T=E_{n} D$ and consider $T$ as an operator in $l_{\infty}^{n}$.

Since $E_{n}$ is a unitary matrix, we conclude that

$$
\prod_{k=1}^{n}\left|\lambda_{k}(T)\right|=|\operatorname{det}(T)|=|\operatorname{det}(D)|=\prod_{k=1}^{n} \sigma_{k} .
$$

For $k=1, \ldots, n$, let $A_{k}$ be the matrix which coincides with $E_{n} D$ on the first $k-1$ columns and has only zero entries in the remaining columns. Obviously, $\operatorname{rank}\left(A_{k}\right)<k$. Hence

$$
a_{k}(T) \leq\left\|T-A_{n}\right\|=\sum_{l=k}^{n} n^{-1 / 2} \sigma_{l} .
$$

Inequality (5) used for our example operator $T$ shows that

$$
\prod_{k=1}^{n} \sigma_{k} \leq c_{n} n^{-n / 2} \prod_{k=1}^{n} \sum_{l=k}^{n} \sigma_{l} .
$$

Since this holds for all $\sigma_{1}>\sigma_{2}>\ldots>\sigma_{n}>0$, a final glimpse at the above lemma proves that $c_{n} \geq n^{n / 2}$.

Remark. In a subsequent paper of B. Carl and the author CH04 we further investigate optimal inequalities of Weyl type. In particular, we show a scale of Weyl type inequalities useful for estimating single eigenvalues with the help of $s$-numbers. We also show that the Weyl numbers are, in some sense, optimal $s$-numbers for inequalities of the type (2) and (3).

\section{REFERENCES}

[CH04] B. Carl, A. Hinrichs, Optimal Weyl type inequalities for operators in Banach spaces. To appear in Positivity.

[Koe84] H. König, Some inequalities for the eigenvalues of compact operators. In Proc. Oberwolfach Conference General Inequalities 1983, Birkhäuser, Basel, 1984, 213-219. MR0821799 (87g:47032)

[Koe86] H. König, Eigenvalue distribution of compact operators. Operator Theory: Advances and Applications 16, Birkhäuser, Basel, 1986. MR0889455 (88j:47021)

[Koe01] H. König, Eigenvalues of operators and applications. In Handbook of the geometry of Banach spaces, Vol. I, North-Holland, Amsterdam, 2001, 941-974. MR1863710 (2003f:47035)

[Pie66] A. Pietsch, Absolut p-summierende Abbildungen in normierten Rumen. Studia Math. 28 (1966/1967), 333-353. MR0216328 (35:7162)

[Pie74] A. Pietsch, s-numbers of operators in Banach spaces. Studia Math. 51 (1974), 201-223. MR 0361883 (50:14325) 
[Pie80a] A. Pietsch, Weyl numbers and eigenvalues of operators in Banach spaces. Math. Ann. 247 (1980), 149-168. MR0568205 (82i:47073a)

[Pie80b] A. Pietsch, Operator ideals. North Holland, Amsterdam, New York, Oxford, 1980. MR:0582655 (81j:47001)

[Pie87] A. Pietsch, Eigenvalues and $s$-numbers. Cambridge Studies in Advanced Mathematics 13. Cambridge University Press, 1987. MR0890520 (88j:47022b)

[Wey49] H. Weyl, Inequalities between two kinds of eigenvalues of a linear transformation. Proc. Nat. Acad. Sci. U. S. A. 35 (1949), 408-411. MR0030693(11:37d)

Mathematisches Institut, FSU Jena, Ernst-Abbe-Platz 1-3, D-07743 Jena, Germany

E-mail address: hinrichs@minet.uni-jena.de

$U R L$ : www.mathematik. uni-jena.de/ ${ }^{\sim}$ hinrichs 